https://doi.org/10.15407/ujpe64.7.543

V. AUSHEV

On behalf of the ZEUS and H1 collaborations

Taras Shevchenko Kyiv National University

(60, Volodymyrs'ka Str., Kyiv, Ukraine, 01033; e-mail: volodymyr.aushev@gmail.com)

\title{
CHARM AND BEAUTY PRODUCTION \\ CROSS-SECTION MEASUREMENTS IN DEEP INELASTIC ELECTRON-PROTON SCATTERING AT HERA
}

\begin{abstract}
The open charm and beauty production cross-sections in the deep inelastic ep scattering (DIS) at HERA from the H1 and ZEUS Collaborations are combined. Reduced cross-sections are obtained in the kinematic range of negative four-momentum transfer squared of a photon $2.5 \leq Q^{2} \leq 2000 \mathrm{GeV}^{2}$ and the Bjorken scaling variable $3 \times 10^{-5} \leq x_{B j} \leq 5 \times 10^{-2}$. The different charm- and beauty-tagging methods are used for the heavy-flavor production study in DIS. The combined method accounts for the correlations of systematic uncertainties, as well as statistical uncertainties among the different datasets. Perturbative $Q C D(p Q C D)$ calculations are compared to the measured combined data. A NLO QCD analysis is performed using these data together with the combined inclusive deep inelastic scattering crosssections from HERA. The running charm- and beauty-quark masses are determined as $m_{c}\left(m_{c}\right)=1.290_{-0.041}^{+0.046}(\exp / f i t)_{-0.014}^{+0.062}(\text { model })_{-0.031}^{+0.03}($ parametrization $)$ GeV and $m_{b}\left(m_{b}\right)=$ $=4.049_{-0.109}^{+0.104}(\exp / \mathrm{fit})_{-0.032}^{+0.090}(\text { model })_{-0.031}^{+0.001}$ (parametrization) $\mathrm{GeV}$.
\end{abstract}

Keywords: charm and beauty production, deep inelastic interaction, electron-proton scattering, quark mass, perturbative QCD, combined cross-sections.

\section{Introduction}

Measurements of open charm and beauty productions in the deep inelastic ep-scattering at HERA provide the important input for tests of quantum chromodynamics (QCD). HERA collected about $0.5 \mathrm{fb}^{-1}$ of the integrated luminosity by each experiment. Measurements at HERA have shown that the heavy-flavor (HFL) production in DIS proceeds predominantly via the boson-gluon-fusion process, i.e. $\gamma g \rightarrow q \bar{q}$. Therefore, the cross-section depends on the gluon distribution in the proton, as well as the heavy-quark mass. This mass provides a hard scale for the applicability of pQCD. At the same time, other hard scales are also present in this process such as the transverse

(C) V. AUSHEV, 2019

ISSN 2071-0194. Ukr. J. Phys. 2019. Vol. 64, No. 7 momenta of the outgoing quarks and the virtuality, $Q^{2}$, of the exchanged photon. The presence of several hard scales complicates the calculation of the HFL production in pQCD. We used different approaches to cope with the multiple scale problem. In our study, the massive fixed-flavor-number scheme (FFNS) and the variable-flavor-number scheme (VFNS) are used.

The ZEUS and H1 detector systems at the HERA electron-proton collider were general purpose detectors. They have a similar structure and consist of tracking systems (including high-resolution silicon vertex detectors) surrounded by electromagnetic and hadronic calorimeters and muon detectors. This provides almost $4 \pi$ coverage of the collision region.

In this report, a $\mathrm{H} 1$ and ZEUS combination of the charm and beauty quark productions is presented 
[4]. This analysis is an extension of the previous combination of charm cross-section measurements in DIS, including new charm and beauty data. A single consistent dataset of reduced charm and beauty crosssections from both detectors is obtained. All correlations are included. This dataset covers the kinematic range of boson (photon) virtualities $2.5 \leq Q^{2} \leq$ $\leq 2000 \mathrm{GeV}^{2}$ and the Bjorken scaling variable in the region $3 \times 10^{\neg 5} \leq x_{B j} \leq 5 \times 10^{-2}$.

For these measurements, different flavor tagging methods are used: the full reconstruction of $D$ or $D^{\star \pm}$ mesons, which is sensitive to the charm production; the lifetime of heavy-flavored hadrons and their semileptonic decays. This allows the measurement of charm and beauty cross-sections simultaneously. These methods show the dependences on sources of systematic uncertainties for different regions of the heavy-quark phase space. The simultaneous combination of charm and beauty cross-section measurements reduces all uncertainties. The combined charm crosssections of the previous analysis [1] are superseded by the new results presented in this paper. The measured combined beauty cross-sections are presented for the first time. In general, this paper sums up almost 20 years of HFL researches at HERA.

The new data are analyzed using QCD methods for determining the running charm and beauty quark masses at the NLO calculations in the minimumsubtraction (MS) scheme. The FFNS is used for pQCD calculations for the corrections of measurements to the full phase space and in the QCD fits. In this scheme, heavy quarks are always treated as massive. The number of active flavors in the PDFs, $n_{f}$, is set equal to 3 . In this model, heavy quarks are produced only in the hard-scattering ep process. In all FFNS heavy-quark calculations presented in this paper, the default renormalization scale $\mu_{r}$ and factorization scale $\mu_{f}$ are set to $\left.\mu_{r}=\mu_{f}=\sqrt{(} Q^{2}+4 m_{Q}^{2}\right)$, where $m_{Q}$ is a pole or running mass. Predictions from different variants of the VFNS are also compared to the data. In the VFNS, heavy quarks are treated as massive at small $Q^{2}$ up to $Q^{2} \approx O\left(m_{Q}^{2}\right)$ and as massless at $Q^{2} \gg m_{Q}^{2}$, with interpolation prescriptions between the two regimes.

\section{Combined Cross-Sections and QCD Analysis}

The data have been obtained from both the HERA I (in the years 1992-2000) and HERA II (in the years
2003-2007) data-taking periods. The combination includes measurements based on using different HFLtagging techniques: the reconstruction of particular decaying $\mathrm{D}$ mesons, the inclusive analysis of tracks exploiting the lifetime information and the reconstruction of electrons and muons from heavy-quark semileptonic decays.

A total of 209 charm and 57 beauty data points are combined simultaneously to obtain 52 charm and 27 beauty cross-section data-sets. A $\chi^{2}$ value of 149 for 187 degrees of freedom is obtained in the combination, indicating a good consistency of the input data. There are 167 sources of correlated uncertainties in total. These are 71 experimental systematic sources, 16 sources due to the extrapolation procedure (including the uncertainties on the fragmentation fractions and branching ratios), and 80 statistical charm and beauty correlations.

The experiments at HERA typically measure the so-called reduced cross-section, $\sigma_{\text {red }}$, which is closely related to the double-differential cross-section in the kinematic quantities $Q^{2}$ and $x$. The combined reduced cross-sections $\sigma_{\text {red }}^{\text {cc }}$ are shown as functions of $x_{B j}$ in bins of $Q^{2}$ together with the input $\mathrm{H} 1$ and ZEUS data in Fig. 1. As we can see, the combined cross-sections are significantly more precise than any of the individual input data-sets for the charm and beauty productions. This is illustrated in Fig. 2, where the charm measurements for $Q^{2}=32 \mathrm{GeV}^{2}$ are shown. The uncertainty of the combined charm cross-section is $9 \%$ on the average and reaches values of about $5 \%$ or better in the region $12 \mathrm{GeV}^{2} \leq$ $\leq Q^{2} \leq 60 \mathrm{GeV}^{2}$. The uncertainty of the combined beauty cross-section is about $25 \%$ on the average and reaches about $15 \%$ at small $x_{B j}$ and $12 \mathrm{GeV}^{2} \leq Q^{2} \leq$ $\leq 200 \mathrm{GeV}^{2}$.

Theoretical predictions of the FFNS in the MS running mass scheme are compared to the combined reduced cross-sections $\sigma_{\text {red }}^{\text {cc }}$ and $\sigma_{\text {red }}^{\text {bb }}$, as we can see in Figs. 3 and 4, respectively. In these calculations, the running quark masses are set to the world average values [2] of $m_{c}\left(m_{c}\right)=1.27 \pm 0.03 \mathrm{GeV}$ and $m_{b}\left(m_{b}\right)=4.18 \pm 0.03 \mathrm{GeV}$.

The charm cross-sections of the current analysis agree well with the previous measurements, but have considerably smaller uncertainties. The observed changes in the $\chi^{2}$ values are consistent with an improvement in the data precision. The tension observed between the central theory predictions and

ISSN 2071-0194. Ukr. J. Phys. 2019. Vol. 64, No. 7 


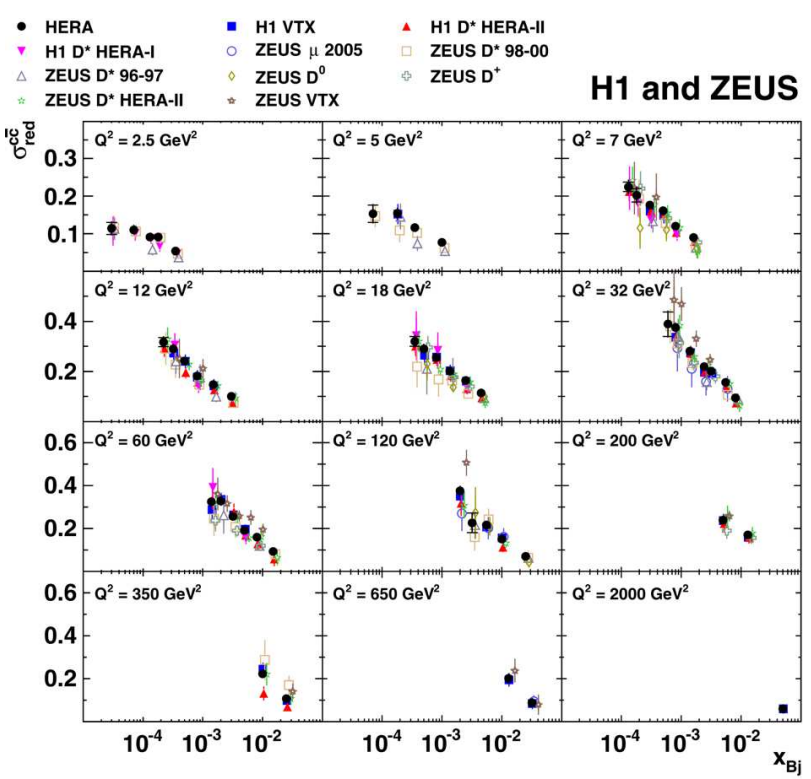

Fig. 1. Combined measurements of the charm production cross-sections, $\sigma_{\text {red }}$, (full circles) as functions of $x_{B j}$ for different values of $Q^{2}$. The inner error bars indicate the uncorrelated part of the uncertainties, and the outer error bars represent the total uncertainties. The input measurements with their total uncertainties are also shown by different markers. For a better visibility, the individual input data are slightly displaced in $x_{B j}$ toward larger values

the charm data ranges from $\sim 3 \sigma$ to more than $6 \sigma$, depending on the prediction. The NLO FFNS calculations provide the best description of the charm data. For the beauty cross-sections, a good agreement of theory and data is observed within the larger experimental uncertainties. The effect of the PDF uncertainties on the $\chi^{2}$ values is negligible.

The combined charm and beauty data are used together with the combined HERA inclusive DIS data [3] to perform a QCD analysis. In our QCD analysis, we determined simultaneously the running heavy-quark masses $m_{c}\left(m_{c}\right)$ and $m_{b}\left(m_{b}\right)$. We investigated the $x_{B j}$ dependence of the reduced charm cross-sections. We used the XFITTER programme, in which the scale evolution of partons is calculated through DGLAP equations at NLO (using the QCDNUM programme). The theoretical FFNS predictions for the HERA data are obtained using the OPENQCDRAD programme interfaced in the XFITTER framework. The number of active flavors is set to $n_{f}=3$ at all scales. For the heavy-quark contributions, the scales are set to $\left.\mu_{r}=\mu_{f}=\sqrt{(} Q^{2}+4 m_{Q}^{2}\right)$.

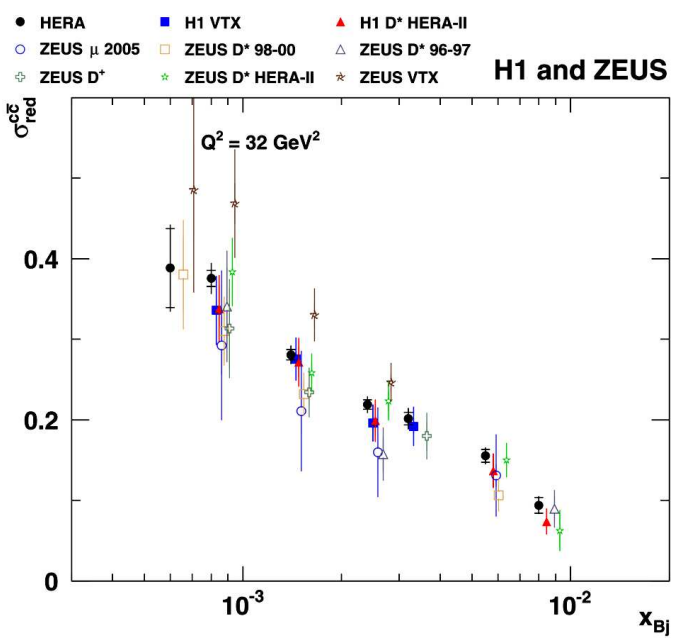

Fig. 2. Reduced cross-sections as a function of $x_{B j}$ at $Q^{2}=32 \mathrm{GeV}^{2}$ for the charm production. The combined crosssections (full circles) are compared to the input measurements shown by different markers. For the combined measurements, the inner error bars indicate the uncorrelated part of the uncertainties and the outer error bars represent the total uncertainties

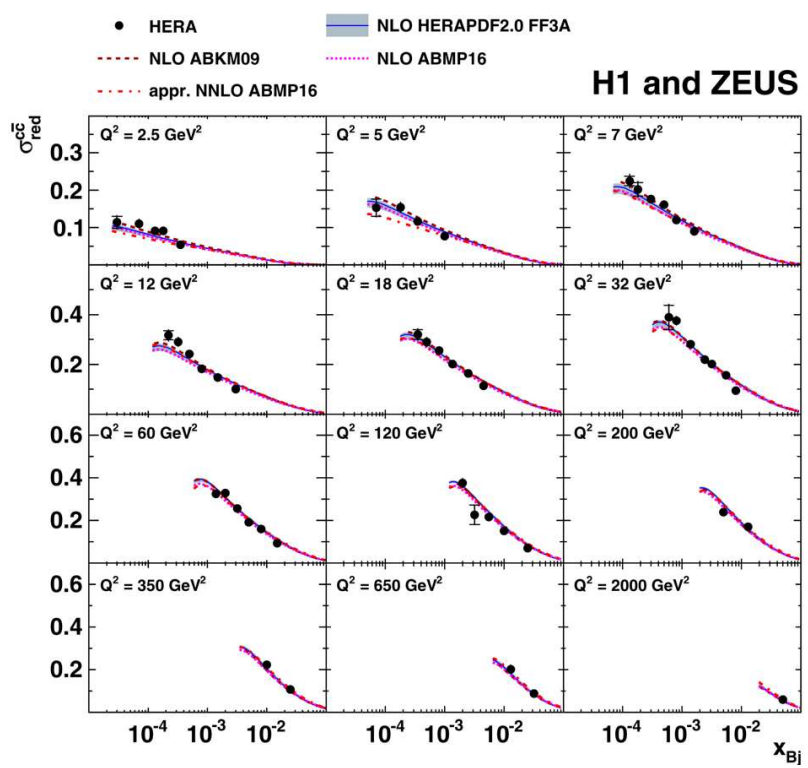

Fig. 3. Combined reduced charm cross-sections, $\sigma_{\text {red }}^{\mathrm{cc}}$ (full circles) as functions of $x_{B j}$ for given values of $Q^{2}$, compared to the NLO QCD FFNS predictions based on the HERAPDF2.0 FF3A (solid lines), ABKM09 (dashed lines), and ABMP16 (dotted lines) PDF sets. The approximate NNLO prediction using ABMP16 (dash-dotted lines) is also shown. The shaded bands on the HERAPDF2.0 FF3A predictions show the theory uncertainties obtained by adding PDF, scale, and charm-quark mass uncertainties in quadrature 


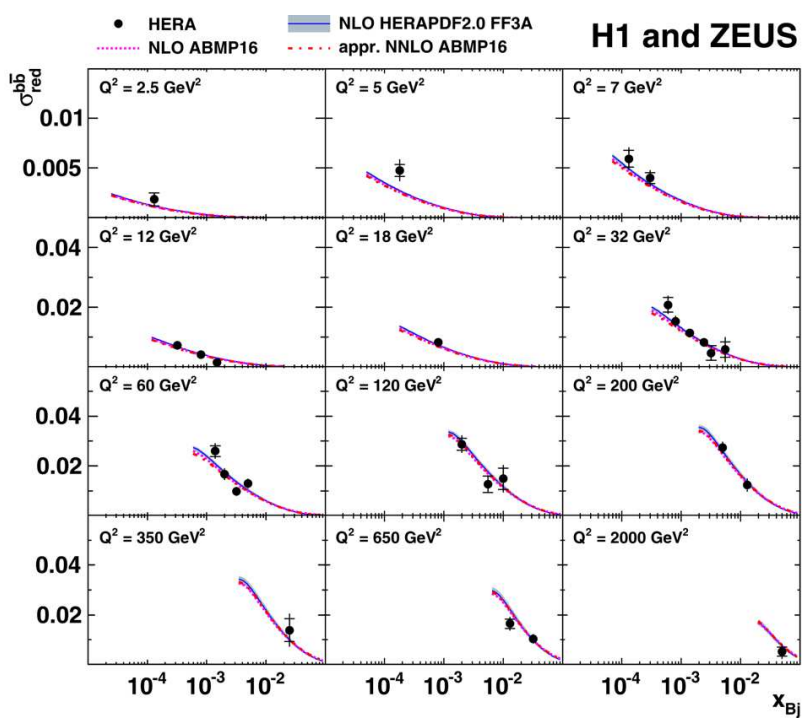

Fig. 4. Combined reduced beauty cross-sections $\sigma_{\text {red }}^{\text {bb }}$ (full circles) as functions of $x_{B j}$ for the given values of $Q^{2}$, compared to the NLO QCD FFNS predictions based on the HERAPDF2.0 FF3A (solid lines), ABKM09 (dashed lines), and ABMP16 (dotted lines) PDF sets. The approximate NNLO prediction using ABMP16 (dashed-dotted lines) is shown as well. The shaded bands on the HERAPDF2.0 FF3A predictions show the theory uncertainties obtained by adding PDF, scale, and beauty-quark mass uncertainties in quadrature

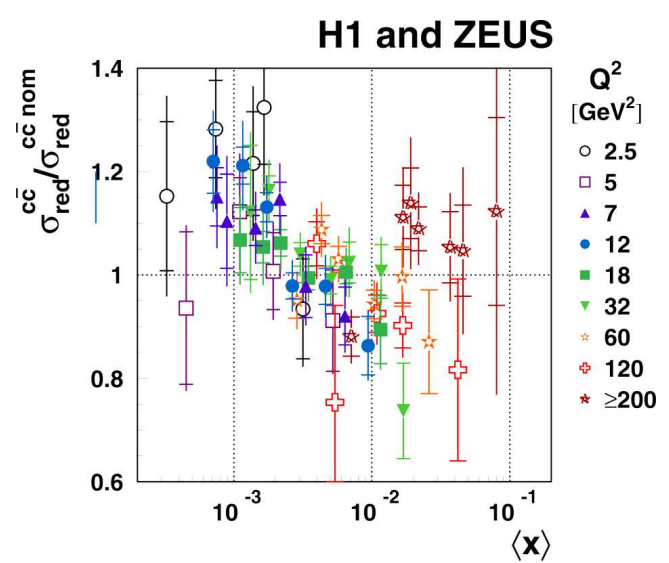

Fig. 5. Ratio of the combined reduced cross-sections, $\sigma_{\text {red }}^{\mathrm{cc}}$ to the respective NLO FFNS cross-section predictions, $\sigma_{\text {red }}^{\text {nom }}$ based on HERAPDF-HQMASS, as a function of the partonic average $x$ for different values of $Q^{2}$

The $\mathrm{c}$ and b-quark masses are left free by fitting. The running heavy-quark masses are fitted simultaneously with the PDF parameters. The fit yields a total $\chi^{2}=$ $=1435$ for 1208 degrees of freedom. The smaller un- certainties of the new combination reduce the uncertainty of the charm-quark mass determination with respect to the previous result. The beauty quark mass determination improves the previous result based on a single dataset. The running quark masses are determined (in $\mathrm{GeV}$ ) as:

$m_{c}\left(m_{c}\right)=$

$=1.290_{-0.041}^{+0.046}(\exp / \text { fit })_{-0.014}^{+0.062}(\operatorname{model})_{-0.031}^{+0.03}($ param $)$,

$m_{b}\left(m_{b}\right)=$

$=4.049_{-0.109}^{+0.104}(\exp / \text { fit })_{-0.032}^{+0.090}(\text { model })_{-0.031}^{+0.001}($ param $)$.

The model uncertainties are dominated. A better description of the charm data can be achieved, if $x_{B j} \leq$ $\leq 0.01$ are excluded from the fit. Alternative NLO and NNLO QCD calculations, including those with low- $x$ resummation, do not provide a better description of the combined heavy-quark data.

Since, in LO QCD, the heavy-quark production proceeds via the photon-gluon-fusion, at least two partons are present in the final state. The $x$ of the incoming parton is different from $x_{B j}$ measured at the photon vertex. The $x$ of the gluon is equal to

$x=x_{B j}\left(1+\frac{\hat{s}}{Q^{2}}\right)$

where $\hat{s}$ is the invariant mass of the heavy-quark pair. In Fig. 5, the ratio of the measured reduced cross-sections to the NLO FFNS predictions based on HERAPDF-HQMASS is shown. More detailed studies of the $x$ slope tension showed that it can not be solved by varying the gluon density, or adding higher orders, or resumming $\log \left(\frac{1}{x}\right.$ terms, within the respective pQCD frameworks.

\section{Conclusions}

The results of measurements of charm and beauty production reduced cross-sections in the deep inelastic ep scattering by the H1 and ZEUS experiments at the HERA collider are combined for the first time (beauty) and significantly reduced uncertainties (charm) than those previously published. Next-toleading and approximate next-to-next-to-leading order QCD predictions are compared to the data. Calculations are found to be in a good agreement with the charm data. The NLO calculations in the fixedflavor-number scheme provide the best description of 
the heavy-flavor data. The beauty data have larger experimental uncertainties. These data are well described by all QCD predictions. The new combined data are subjected to a NLO QCD analysis in the fixed-flavor-number scheme using the MS runningmass definition. The running heavy-quark masses are determined from combined data. The simultaneously determined parton density functions are found to agree well with HERA-PDF2.0 FF3A. The QCD analysis reveals some tensions, at the level of $3 \sigma$, in describing simultaneously the inclusive and heavyquark HERA DIS data. The measured reduced charm cross-sections show a stronger $x_{B j}$ dependence than that obtained in the combined QCD fit of charm and inclusive data, in which the PDFs are dominated by the fit of the inclusive data.

The work was supported by the Ministry of Education and Science of Ukraine

1. H. Abramowicz et al. [H1 and ZEUS Collaborations]. Combination and QCD analysis of charm production cross section measurements in deep-inelastic ep scattering at HERA Eur. Phys. J. C 73, 2311 (2013) [arXiv:1211.1182].

2. C. Patrignani et al. Particle Data Group. Review of particle physics. Chin. Phys. C 40, 100001 (2016).

3. H. Abramowicz et al. [H1 and ZEUS Collaborations]. Combination of measurements of inclusive deep inelastic $e^{ \pm} p$ scattering cross sections and QCD analysis of HERA data. Eur. Phys. J. C 75, 580 (2015) [arXiv:1506.06042].

4. H. Abramowicz et al. [H1 and ZEUS Collaborations]. Combination and QCD analysis of charm and beauty production cross-section measurements in deep inelastic ep scattering at HERA Eur. Phys. J. C 78, 473 (2018).

Received 08.07.19

B. Аушев

ВИМІРЮВАННЯ ПОПЕРЕЧНИХ ПЕРЕРІЗІВ

УTВOРЕННЯ KBAPKIB CHARM TA BEAUTY

В ЕЛЕКТРОН-ПРОТОННИХ

ЗITKHEHНЯX HA HERA

Р е $з$ ю м е

Наведено комбіновані значення перерізів утворення $c$ та $b$ кварків в глибоко-непружних електрон-протонних взаємодіях на колайдері HERA, виміряних колабораціями ZEUS i H1. Параметри визначено в діапазоні віртуальності обмінного фотона $2,5 \leq Q^{2} \leq 2000 \Gamma^{2} \mathrm{~B}^{2}$ та значення змінної Бйоркена $3 \cdot 10^{\neg 5} \leq x_{B j} \leq 5 \cdot 10^{\neg 2}$. Використано різні методи тагування $c$ та $b$ кварків, які спираються на всебічне дослідження важких ароматів. При комбінуванні обчислювались статистичні та систематичні невизначеності для різних наборів даних. Для обчислень використовувалась пертурбативна КХД в різних порядках наближення і результати обчислень порівнювались з виміряними даними. Визначено поточні значення мас $c$ та $b$ кварків, які становлять: $m_{c}\left(m_{c}\right)=1,290_{-0,041}^{+0,046}(\exp / \mathrm{fit})_{-0,014}^{+0,062}(\operatorname{model})_{-0,031}^{+0,03}($ param $)$, $m_{b}\left(m_{b}\right)=4,049_{-0,109}^{+0,104}(\exp / \mathrm{fit})_{-0,032}^{+0,090}(\operatorname{model})_{-0,031}^{+0,001}($ param $)$. 